\title{
Spin Wave Excitations of the Interacting Two-Dimensional In-Plane Nano-Vortices
}

\author{
S. MAMICA* \\ Faculty of Physics, A. Mickiewicz University in Poznań, ul. Umultowska 85, 61-614 Poznań, Poland \\ The aim of this work is to study spin-wave excitations in the row of interacting two-dimensional nanodots in \\ the vortex state. We use a discrete dipole model taking into account the nearest-neighbour exchange and dipolar \\ interactions. Magnetic configuration of each dot is assumed to form an in-plane vortex (circular magnetization). \\ We examine the dependence of frequencies and profiles of spin-wave modes vs. the dipolar-to-exchange interaction \\ ratio and the dot separation. Special attention is paid to some particular modes: lowest-frequency azimuthal modes \\ and the fundamental mode, an analogue of the uniform excitation. Some conclusions regarding the influence of \\ the size of dots the row consists of as well as the chirality of neighbouring vortices are provided too.
}

DOI: 10.12693/APhysPolA.133.505

PACS/topics: 75.30.Ds, 75.70.Kw, 75.75.Jn

\section{Introduction}

In magnetic systems the concurrence of short and long range interactions is responsible for a variety of interesting effects [1-3]. In small magnetic dots the competition between the exchange and dipolar interactions leads to a rich spectrum of magnetic configurations including vortices [4]. In potential applications of magnetic vortices, which rage from information processing to microwave-frequency multiplication [5-7], an important role is played by spin waves. In particular, the lowest mode plays a special role in metastable vortices, in which it becomes a soft mode responsible for the transition to different magnetic configurations [8]. This lowest mode is reported to be of different kind, from an azimuthal mode of different order to localized or fundamental one [9-11]. Spin wave excitations are involved in the vortex core switching in an isolated dot [12] but above effects should be visible also in arrays of interacting vortices [13] and vortex crystals [14].

In the present study we focus on the spin-wave spectrum of the row of two-dimensional (2D) circular dots coupled by dipolar interactions. We analyze the formation of the bands and the influence of the dipolar-toexchange interaction ratio, the dot size, and the vortex chirality on the band width. Particular attention is paid on two kind of modes: azimuthal modes of the lowest frequencies and the fundamental mode, a counterpart of the uniform excitation [15].

\section{The row of dots}

The object of our study is a row of circular dots cut out from a 2D square lattice with elementary magnetic moments (spins) in its sites. The diameter of the dot means the diameter of a circle used for cutting out the

\footnotetext{
* corresponding author; e-mail: mamica@amu.edu.pl
}

dot expressed in the unit of the lattice constant $a$. The dots separation $\Delta$, i.e. the distance between neighbouring dots, is measured between two closest sites belonging to these dots, and is constant in the row. For every dot in the row its magnetization is assumed to form an inplane vortex. Since the vortex chirality, i.e. the direction of the in-plane magnetization, can be clockwise or anticlockwise we consider two cases: with the same chirality for all dots in the row and with alternating chirality, where the direction of in-plane magnetization is opposite in neighbouring dots.

We consider the dynamics of a magnetic moment $\boldsymbol{M}_{\boldsymbol{R}}, \boldsymbol{R}$ being the position vector, in the linear approximation, assuming $\left|\boldsymbol{m}_{\boldsymbol{R}}\right| \ll \boldsymbol{M}_{\boldsymbol{R}}|,| \boldsymbol{M}_{0, \boldsymbol{R}}|\approx| \boldsymbol{M}_{\boldsymbol{R}} \mid$ and $\boldsymbol{m}_{\boldsymbol{R}} \perp \boldsymbol{M}_{\boldsymbol{R}}$ where $\boldsymbol{M}_{0, \boldsymbol{R}}$ and $\boldsymbol{m}_{\boldsymbol{R}}$ are the static and dynamic component of the magnetic moment, respectively. To describe the time evolution of $\boldsymbol{m}_{\boldsymbol{R}}$, oscillating harmonically with a frequency $\omega$, we use the damping-free Landau-Lifshitz (LL) equation. In the effective field we take into account the dipolar and exchange interactions. Linearization of the set of LL equations for all magnetic moments in the system leads to the eigenvalue problem for so-called dynamical matrix (see $[15,16]$ for more details). Numerical diagonalization of this matrix yields the frequency spectrum of the spin-wave excitations, and the spin-wave profiles, i.e., the distribution of the in-plane $\left(m_{r}\right)$ and out-of-plane $\left(m_{k}\right)$ amplitudes of precession of the elementary magnetic moments.

The only one material parameter in our model is the dipolar-to-exchange interaction ratio defined as:

$$
d=\frac{\left(g \mu_{\mathrm{B}}\right)^{2} \mu_{0}}{8 \pi a^{3} J}
$$

where $g$ is the $g$-factor, $\mu_{\mathrm{B}}$ the Bohr magneton, $\mu_{0}$ the vacuum permeability, $a$ the lattice constant, and $J$ the nearest-neighbour exchange integral.

Without performing any simulations the information about the stability of the assumed magnetic configuration is received from the spin wave spectrum. The occurrence of zero-frequency excitations (nucleation modes) 
is indicative of the instability of the assumed magnetic state while the lack of nucleation modes implies its (meta)stability.

\section{Results}

First of all, let us recall briefly these properties of spin waves in a single dot which are important for the current work. According to the spin wave profile symmetry, the modes can be described by azimuthal $(m)$ and radial numbers $(n)$, i.e. the numbers of the nodal lines in the particular direction. If the azimuthal number differs from zero there is a pair of modes with $+m$ and $-m$ but in our study it is not necessary to distinguish them so the statement $(n, m)$ means any of two modes: $(n,+m)$ or $(n,-m)$. Since the dot is based on the discreet lattice a frequency splitting appears for pairs of modes with particular azimuthal numbers: if the symmetry of the spin wave profile matches the symmetry of the lattice. In the case of a square lattice it happens for $m$ being the even number while for odd $m$ both modes in the pair have the same frequency [15].

Typical behaviour of spin wave profiles in the row of interacting dots is given in Fig. 1 for an exemplary dots of $L=31$ and $d=0.2$. In Fig. 1a we show spin wave profiles of two degenerate first order azimuthal modes in an isolated dot (the lowest frequency modes in the spectrum).

In Fig. 1b the profiles of $(0,1)$ modes for a row of three dots with the separation $\Delta=8$ and the same chirality are shown. The mode $i=1$ existing in the isolated dot splits into three modes $i=1, i=4$, and $i=6$. For the lowest mode $(i=1)$ excitations in single dots have the same phase while for the highest mode $(i=6)$ the phase in neighbouring dots is opposite. In both modes excitation of the internal dot is stronger then outer ones. For the middle-frequency mode $(i=4)$ there is almost no excitation in the internal dot. This means that the energy of the spin wave is located in outer dots. Excitations in outer dots are in anti-phase. Similarly, mode $i=2$ splits into $i=2, i=3$, and $i=5$ but with the lowest mode in phase and the highest one in anti-phase.

Exactly the same types of modes appear in the row with alternating chirality but the mode order is reversed.

In Fig. 2 we study the dependence of the spin wave frequencies on the dots separation $\Delta$ in the row of three dots of $L=31$ (the same as in Fig. 1) for two values of the dipolar-to-exchange interaction ratio $d$. The behaviour of seven modes is shown: pairs of the 1st, 2nd, and 3rd order azimuthal modes and the fundamental mode $(0,0)$. Solid lines stand for the same chirality of all dots in the row while doted ones for the row with alternating chirality. The frequency of the spin wave of the isolated dot (dashed lines) splits into three frequencies due to the dipolar interaction between dots forming a band. (Please notice that in the model used the exchange interaction is taken only between nearest neighbours in the lattice so any separation larger then a single lattice constant breaks the exchange interaction.) In the bands with odd (a) single dot
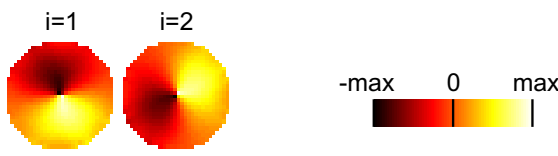

(b) the same chirality

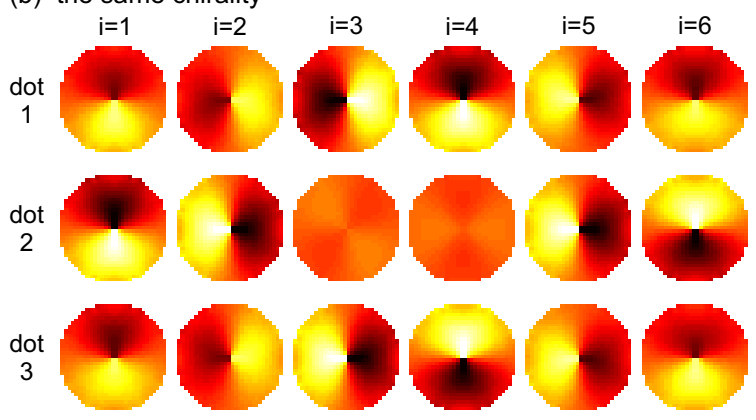

Fig. 1. Spin wave profiles of first order azimuthal modes $(0,1)$ for dots of $L=31$ and $d=0.2$ : (a) an isolated dot, (b) the row of three dots with the same chirality and the dots separation $\Delta=8$. The ' $i$ ' denotes the order of modes in the frequency spectrum.

(a) $d=0.2$

(b) $d=0.5$

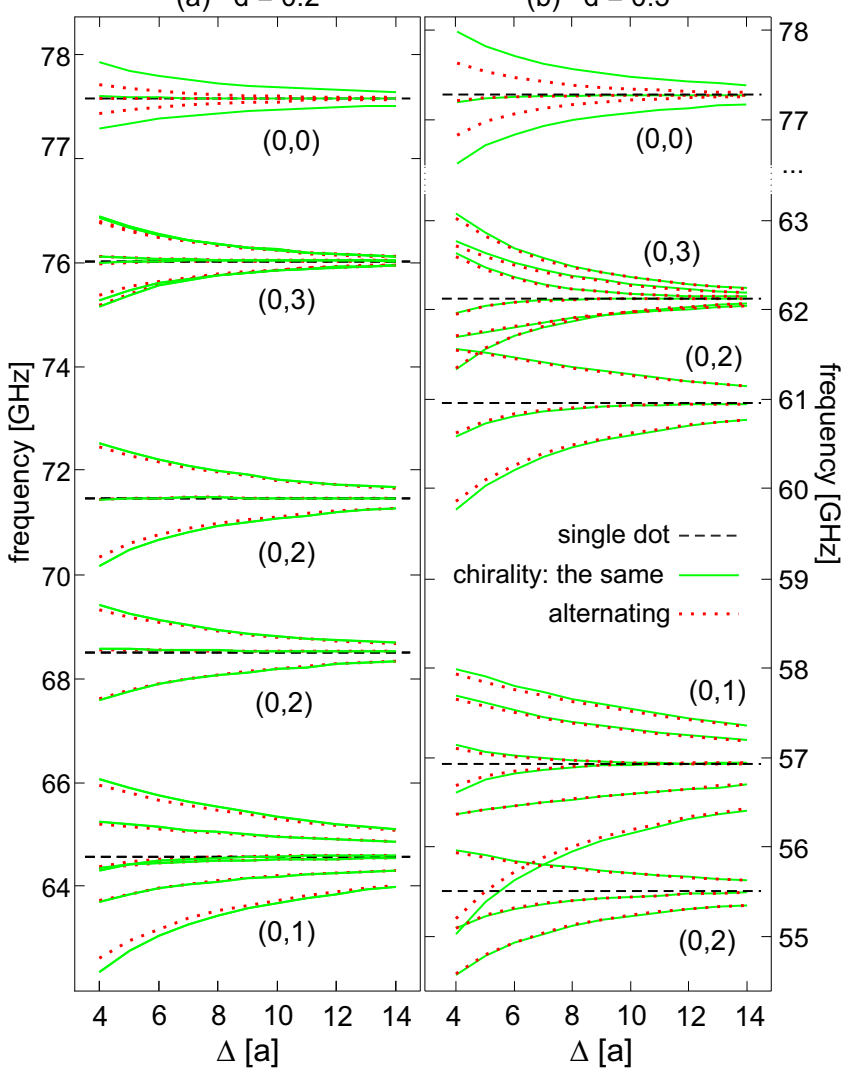

Fig. 2. Evolution of spin wave frequencies vs. dots separation $\Delta$ (in the unit of the lattice constant $a$ ) for the row of three dots of the diameter $L=31$ with the same (solid lines) and alternating (dotted lines) chirality for (a) $d=0.2$ and (b) $d=0.5$. Dashed lines mark frequencies in the isolated dot. 
azimuthal numbers there are six modes because in the isolated dot such modes are degenerate in pairs. Evennumber azimuthal modes form separate bands due to the frequency splitting in the isolated dot. There is only tiny difference in the bands formation for rows with the same and alternating chirality for azimuthal modes regardless even or odd. In contrary, the band formed from a $(0,0)$ mode is few times wider for the same chirality than for alternating one.

For the case of $d=0.2$ (Fig. 2a) first band is formed from two degenerate $(0,1)$ modes, next we have two bands each formed from one of two $(0,2)$ modes, and the fourth band formed from two degenerate $(0,3)$ modes. All these bands are well separated in the range of the distance between dots considered here.

In isolated dots for the majority of modes in the spin wave spectrum the frequency decreases with increasing the dipolar-to-exchange interaction ratio (i.e. weakening of the exchange interaction within the dot) but with different rate depending on the azimuthal number [15]. As a result, the order of modes in the spectrum changes. For the dot in question $(L=31)$ for $d>0.4$ the frequency of one of $(0,2)$ modes starts to be the lowest. Moreover, the distance between frequencies of different modes is smaller. This leads to additional effects in the spectrum of the row of dots.

As an example, in Fig. $2 \mathrm{~b}$ we show the case for $d=0.5$. The change of the band width is similar to the case of $d=0.2$ shown in Fig. 2a (please notice that the scale for the frequencies is different in figures (a) and (b) because of it the band width in (b) appears larger). The lowest band is formed from $(0,2)$ mode and for $\Delta=7$ and below crosses with the second band formed from two degenerated $(0,1)$ modes. Similarly, the third band crosses with the fourth one for $\Delta<5$. Again, the $(0,0)$ band is more than two times wider for the same chirality than for alternating one.

The same behaviour we found for rows of bigger dots but here the crossing of bands occurs for smaller values of $d$ which is caused by the general tendency of the dipolar interaction to favour higher order azimuthal modes regardless of the source of the dipolar interaction strength: the size $(L)$ or material $(d)$ of the $\operatorname{dot}[15]$.

\section{Conclusion}

In this work we study the band formation in the row of three interacting two-dimensional nano-dots in the inplane vortex state. In the range of the dots separation considered here the interaction between dots in the row is only dipolar. For such system any mode existing in an isolated dot splits into three. Two of these modes are excited mostly in the middle dot. The third one is excited almost solely in outer dots - there is no precession of the magnetic moments in the middle dot. This behaviour does not depend on the symmetry of the mode profile nor the chirality of the neighbouring vortices but the change of the chirality changes the mode order.

For small values of the dipolar-to-exchange interaction ratio $d$ the bands are well separated. With growing $d$ the frequency of modes of the isolated dot decreases with the rate depending on the azimuthal number which leads to the change of the mode order. In the row of dots this results in the band crossing if $d$ is large enough. For dots of larger diameter the value of $d$ for which this crossing appears is smaller which reflects the dependence of the dipolar interaction significance on the size of the system.

For azimuthal modes the chirality of neighbouring vortices very little affects the bands formation. The situation is much different for the band formed from the fundamental excitation where this influence is pretty large.

\section{Acknowledgments}

The project leading to these results has received funding from the European Union's Horizon 2020 research and innovation programme under the Marie SkłodowskaCurie grant agreement No 644348 (MagIC).

\section{References}

[1] S. Mamica, H. Puszkarski, J.-C. Lévy, Phys. Status Solidi B 218, 561 (2000).

[2] M. Krawczyk, H. Puszkarski, J.-C. Lévy, S. Mamica, D. Mercier, J. Magn. Magn. Mater. 246, 93 (2002).

[3] J. Romero-Vivas, S. Mamica, M. Krawczyk, V.V. Kruglyak, Phys. Rev. B 86, 144417 (2012).

[4] K. L. Metlov, Y. P. Lee, Appl. Phys. Lett. 92, 112506 (2008).

[5] R. P. Cowburn, M. E. Welland, Science 287, 1466 (2000).

[6] M. Donolato, M. Gobbi, P. Vavassori, M. Leone, M. Cantoni, V. Metlushko, B. Ilic, M. Zhang, S. X. Wang, R. Bertacco, Nanotechnology 20, 385501 (2009).

[7] V. E. Demidov, H. Ulrichs, S. Urazhdin, S. O. Demokritov, V. Bessonov, R. Gieniusz, A. Maziewski, Appl. Phys. Lett. 99, 012505 (2011).

[8] S. Mamica, J.-C. S. Lévy, Ph. Depondt, M. Krawczyk, J. Nanopart. Res. 13, 6075 (2011).

[9] M. Buess, T. P. J. Knowles, R. Hollinger, T. Haug, U. Krey, D. Weiss, D. Pescia, M. R. Scheinfein, C. H. Back, Phys. Rev. B 71, 104415 (2005).

[10] X. Zhu, Z. Liu, V. Metlushko, P. Grutter, M. R. Freeman, Phys. Rev. B 71, 180408(R) (2005).

[11] R. Wang, X. Dong, Appl. Phys. Lett. 100, 082402 (2012).

[12] Ph. Depondt, J.-C. S. Lévy, S. Mamica, J. Phys.: Condens. Matter 25, 466001 (2013)).

[13] M.-Y. Im, P. Fischer, H.-S. Han, A. Vogel, M.-S. Jung, W. Chao, Y.-S. Yu, G. Meier, J.-I. Hong, K.-S. Lee, NPG Asia Mater. 9, e348 (2017).

[14] M. Hänze, Ch. F. Adolff, B. Schulte, J. Möller, M. Weigand, G. Meier, Sci. Rep. 6, 22402 (2016).

[15] S. Mamica, J.-C. S. Lévy, M. Krawczyk, J. Phys. D 47, 015003 (2014).

[16] S. Mamica, in Vortex Dynamics and Optical Vortices, Ed. Hector Pérez-de-Tejada, InTech, Rijeka 2017, p. 317. (open access). 